\title{
NEPHROGENIC DIABETES INSIPIDUS (NDI): CLINICAL, LABORATORY AND GENETIC CHARACTERIZATION OF FIVE BRAZILIAN PATIENTS
}

\author{
Maria Helena Vaisbich, I Juliana Carneiro, ${ }^{\text {I }}$ Wolfanga Bóson, ${ }^{I I}$ Bruna Resende, ${ }^{\text {II }}$

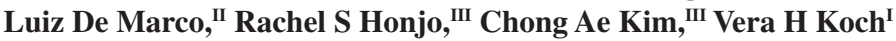

doi: $10.1590 / \mathrm{S1807-59322009000500007}$

Vaisbich MH, Carneiro J, Bóson W, Resende B, De Marco L, Honjo RS et al. Nephrogenic diabetes insipidus (NDI): clinical, laboratory and genetic characterization of five Brazilian patients. Clinics. 2009;64(5):409-14.

INTRODUCTION: Nephrogenic diabetes insipidus is characterized by a lack of response in the distal nephron to the antidiuretic hormone arginine vasopressin. Manifestations include polyuria, polydipsia, hyposthenuria, recurrent episodes of dehydration and fever and growth failure. Most cases are caused by mutations in the AVPR2 gene. The mutant receptors are trapped intracellularly.

METHOD: We studied five boys using clinical, laboratory and molecular data. The mean age at diagnosis was 14.6 months (range 6 to 24$)$ and 12.2 years ( 7.8 to 19$)$ after the follow-up period. The mean period of follow-up was $132.2 \pm 50.9$ months.

RESULTS: The geometric means of the z-scores of weight and stature were -4.5 and -3.6 , respectively, at diagnosis. At the last medical appointment, the z-scores of weight and stature were -0.3 and -0.9 , respectively. Three patients were diagnosed with ureterohydronephrosis and exhibited increased post-void urine volume. Mutations in the AVPR2 gene were found in all patients, and the carrier status was confirmed in four of five cases. Two unrelated children presented identical mutations (S167L) in arginine vasopressin R2. Two of the patients had a mutation that has already been described in other Brazilian families (R337X), and one patient showed a de novo mutation (Y128D) in arginine vasopressin R2, since his mother's molecular analysis was normal. The recurrence risk for this family was significantly reduced.

CONCLUSION: This study reports the clinical and laboratory characterization of Nephrogenic diabetes insipidus and reiterates the importance of the genetic basis that underlies the disease diagnosis and genetic counseling.

KEYWORDS: Nephrogenic insipidus diabetes; Children; Molecular study; Pharmacological chaperones; Hydrochlorothiazide.

\section{INTRODUCTION}

Nephrogenic diabetes insipidus (NDI) is characterized by a lack of response of the distal nephron to the antidiuretic hormone AVP (arginine vasopressin). As a consequence, the kidneys fails to concentrate urine. ${ }^{1,2} \mathrm{NDI}$, which can be either inherited or acquired, is clinically indistinguishable from the central diabetes insipidus. The diagnosis of NDI is confirmed

\footnotetext{
${ }^{\text {I }}$ Unidade de Nefrologia Pediátrica, Instituto da Criança, Hospital das Clínicas da Faculdade de Medicina da Universidade de São Paulo - São Paulo/SP, Brazil.

II Departamento de Cirurgia, Faculdade de Medicina, Universidade Federal de Minas Gerais - Belo Horizonte/MG, Brazil.

III Unidade de Genética, Instituto da Criança, Hospital das Clínicas da Faculdade de Medicina da Universidade de São Paulo - São Paulo/SP, Brazil. Email: maria.vaisbich@icr.usp.br

Tel.: 55113069.8585

Received for publication on December 02, 2008

Accepted for publication on February, 03, 2009
}

by the water deprivation test under 1-desamino-8-D-arginine vasopressin (DDAVP) that demonstrates a lack of increase of urine osmolality and no reduction in urine volume or free water clearance. ${ }^{3}$

The hereditary form of NDI is a rare condition that develops soon after birth and can present as a failure to thrive accompanied by mental retardation, especially if diagnosis and appropriate treatment are delayed. ${ }^{1,2}$ The most common manifestations are polyuria, polydipsia and hyposthenuria with recurrent episodes of dehydration and fever, along with poor nutritional intake and growth failure.

The antidiuretic hormone 8-arginine vasopressin $(\mathrm{AVP})^{3,4}$ binds to a specific receptor, the vasopressin V2 receptor (AVPR2), located on the basolateral membrane of the collecting duct cells. ${ }^{2}$ A cascade of reactions then takes place: the receptor-linked protein $\mathrm{G}$ activation, adenyl cyclase activation, cyclic AMP (cAMP) production and 
protein kinase stimulation. Finally, there is an increase in the exocytic insertion of specific water channels, namely aquaporin 2 or AQP2, into the luminal membrane that ultimately results in increased water permeability. ${ }^{2,4,5}$ In summary, AVPR2 and AQP2 are critical compounds for the transepithelial water permeability of the principal cells of the collecting ducts because a loss of function of either of these proteins will result in NDI (Figure 1).

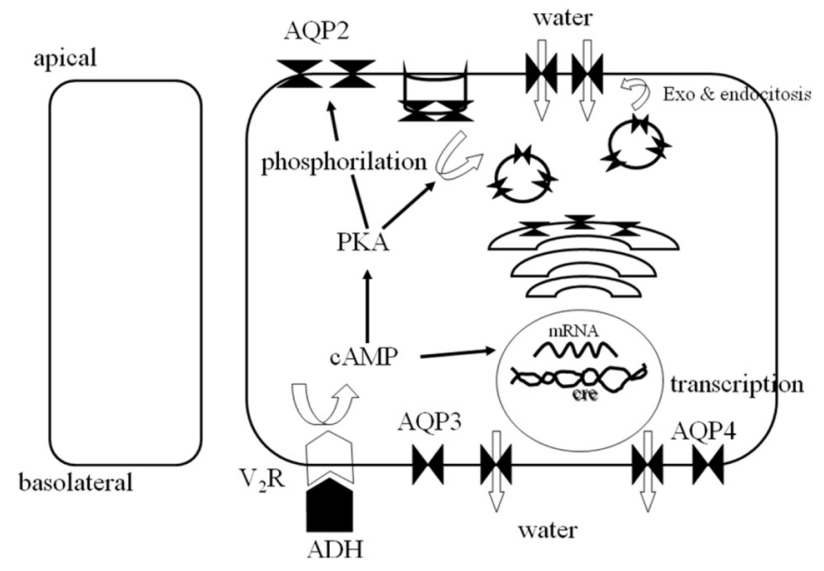

Figure 1 - Illustrative model of the arginine vasopressin (AVP) action in a collecting tubular cell, showing the AVP binding to V2 receptor (V2R), causing the deflagration of a cascade of events. The final result is the migration of water channels (AQP2) to the luminal surface with consequent water reabsorption

In the majority of cases (approximately 90\%), ${ }^{1}$ the defect is secondary to a mutation in the $A V P R 2$ gene, ${ }^{6}$ resulting in an $\mathrm{X}$-linked recessive inheritance pattern. Thus, this form of NDI occurs mainly in males, although there have been reports of heterozygote females with variable manifestations of the disease. ${ }^{7}$ The AVPR 2 gene is located in chromosome region Xq28 and features three exons and two small introns. The sequence of the complementary DNA predicts a polypeptide of 371 amino acids with seven transmembrane, four extracellular, and four cytoplasmic domains. ${ }^{3,8} \mathrm{To}$ date, approximately 190 mutations have been described worldwide. ${ }^{9}$

The autosomal forms of inheritance, which are much less frequent, are due to mutations in the $A Q P 2$ gene., 3,6 The human $A Q P 2$ gene is located in chromosome region $12 \mathrm{q} 13$, features four exons and three introns, and codes for a polypeptide of 271 amino acids. ${ }^{10,11}$

Most missense AVPR2 mutations lead to receptors that are trapped intracellularly; a few mutant receptors reach the cell surface but are unable to bind AVP or to properly trigger an intracellular cyclic adenosine monophosphate signal ${ }^{3}$. Similarly, most $A Q P 2$ mutant proteins are also misrouted. ${ }^{11}$ As numerous studies have revealed that endoplasmic reticulum (ER)-retained mutant proteins are often functional, research over the past decade has focused on identifying compounds that can rescue the cell surface expression of such proteins. In this respect, the vasopressin type 2 receptor $(\mathrm{V} 2 \mathrm{R})$ is the prototypical protein, as it was the first receptor for which the exciting discovery was made that cell-permeable antagonists (CPAn, known as "pharmacochaperones") can promote cell surface trafficking of its ER-retained mutants. ${ }^{12}$ Studies with these antagonists have been performed in the context of NDI. ${ }^{13}$

Since there have been meaningful advances with the development of pharmacological chaperones, Fujiwara and Bichet ${ }^{10}$ suggest that all families with NDI should undergo molecular analysis because of the availability of immediate clinical and early therapeutic measures, and the possibility of preventing recurrent dehydration episodes and their consequences.

This study was performed to report the clinical, laboratory and genetic characteristics of patients with NDI, from the Pediatric Nephrology Unit of the Instituto da Criança - HCFMUSP.

\section{MATERIAL AND METHODS}

Clinical and laboratory data from patients with NDI, followed in the outpatient Pediatric Nephrology Unit of the Instituto da Criança - HCFMUSP, were recorded in medical records at the time of diagnosis and at the last medical appointment. The data consisted of demographic factors (age, gender, and race); anthropometric measurements (weight and height); clinical manifestations and physical exam at diagnosis, including hydration status and irritability; familial history; and the degree of compromise of extra-renal organs. In the follow-up period, we observed the growth parameters (weight and height) and the neurological status (developmental milestones, neurological sequelae).

The following blood tests were performed: sodium, potassium, chloride, urea, creatinine, plasma osmolality and venous blood gas. In addition, urine exams recorded $\mathrm{pH}$, density, osmolality, sodium, potassium, chloride and the presence of proteinuria. An annual renal ultrasonography was also included, mainly to evaluate urinary tract dilatation and post-void urine volume.

The molecular analysis was performed at the Molecular Genetics Laboratory - Faculdade de Medicina da Universidade Federal de Minas Gerais (UFMG), Brazil, using PCR amplification with specific primers followed by direct sequencing of the AVPR2 gene using $\mathrm{ABI} 310$ and $\mathrm{ABI}$ 3130 Genetic Analyzers (Applied Biosystems, Foster City, CA) as previously described. ${ }^{9,14}$ Peripheral blood samples from the patient and the parents were collected, and genomic DNA were extracted for analysis using the GenomicPrep 
Table 1 - Summarized data from the five NDI patients

\begin{tabular}{lccccccc}
\hline Patient & Gender & $\begin{array}{c}\text { Age at diagnosis } \\
\text { (months) }\end{array}$ & $\begin{array}{c}\text { Age at final visit } \\
\text { (years) }\end{array}$ & $\begin{array}{c}\text { Z-score of } \\
\text { initial weight }\end{array}$ & $\begin{array}{c}\text { Z-score of final } \\
\text { visit weight }\end{array}$ & $\begin{array}{c}\text { Z-score of } \\
\text { initial stature }\end{array}$ & $\begin{array}{c}\text { Z-score of final } \\
\text { visit stature }\end{array}$ \\
\hline 1 & Male & 24 & 15 & -4.73 & 0.63 & -3.73 & -0.58 \\
2 & Male & 20 & 19 & -3.61 & -0.08 & -3.50 & -0.20 \\
3 & Male & 15 & 7.8 & -3.56 & -0.37 & -2.97 & -1.44 \\
4 & Male & 6 & 10.4 & -5.55 & -1.81 & -4.54 & -2.43 \\
5 & Male & 8 & 9 & -5.48 & -0.10 & -3.33 & 0.06 \\
\hline
\end{tabular}

Table 2 - Laboratory findings

\begin{tabular}{|c|c|c|c|c|c|c|}
\hline \multirow[t]{2}{*}{ Patient } & \multicolumn{2}{|c|}{ Serum creatinine (mg/dL) } & \multicolumn{2}{|c|}{ Serum urea $(\mathrm{mg} / \mathrm{dL})$} & \multicolumn{2}{|c|}{ creatinine clearance $\left(\mathrm{ml} / \mathrm{min} / 1.73 \mathrm{~m}^{2} \mathrm{bs}\right)$} \\
\hline & at presentation & at final visit & at presentation & at final visit & at presentation & at final visit \\
\hline 1 & 0.8 & 0.95 & 41 & 22 & 43.6 & 126.7 \\
\hline 2 & 0.7 & 1.2 & 38 & 30 & 48.2 & 99.1 \\
\hline 3 & 0.26 & 0.52 & 13.5 & 31 & 121.1 & 108.1 \\
\hline 4 & 0.53 & 0.39 & 41 & 10 & 49.25 & 150.5 \\
\hline 5 & 0.6 & 0.36 & 49 & 17 & 47.2 & 160.0 \\
\hline
\end{tabular}

BS = body surface

Blood DNA Isolation kit (GE HealthCare, Piscataway, NJ) following the manufacturer's recommended protocol. All PCR products were sequenced in both forward and reverse, in duplicate.

Whenever possible, we calculated the geometric means and standard deviations, as well as the z-scores for stature and weight.

\section{RESULTS}

Five boys with NDI were studied. The age at diagnosis ranged from 6 to 24 months (mean $=14.6$ months) and from 7.8 to 19 years (mean $=12.2$ years) after the followup period. The mean period of follow-up was $132.2 \pm 50.9$ months.

All patients presented with failure to thrive, fever and dehydration episodes, polyuria and polydipsia. In addition, they had each been admitted to the hospital several times prior to diagnosis. In all cases, NDI was confirmed using the water deprivation test which showed a lack of response to DDAVP.

Table 1 shows the gender, age at diagnosis and at last appointment, and z-scores for weight and height at diagnosis and at the end of the study for each patient. The geometric mean of the z-score was -4.5 for weight and -3.6 for stature at diagnosis. At the last visit, the $\mathrm{z}$-score was -0.3 for weight and -0.9 for stature. Table 2 shows the laboratory results at diagnosis and at the last appointment. The glomerular filtration rate (GFR) was estimated using the Schwartz equation. Table 3 lists the neurological findings of the five patients studied.

Table 3 - Neurological findings

\begin{tabular}{lc}
\hline Patient & Neurological abnormalities \\
\hline 1 & Cognitive compromise \\
2 & None \\
3 & None \\
4 & None \\
5 & Seizures \\
\hline
\end{tabular}

Among all five patients, three were diagnosed with ureterohydronephrosis and increased post-void urine volume. Such finding was more common in those patients with a longer disease profile.

The chosen therapeutic approach in all cases included an increase in water intake and a low-salt, normal protein diet $(2-2.5 \mathrm{mg}$ of protein per kilogram daily). All patients were administered hydrochlorothiazide (1-2 mg/kg/day); three patients needed potassium replacement for hypokalemia during the treatment, and only one patient (patient 2) used a non-steroidal anti-inflammatory drug (Celecoxib®) for 15 days in an effort to reduce the enormous urinary volume. However, this patient subsequently developed arterial hypertension, which was controlled after use of this drug 
was discontinued. Patient 5 presented a severe hemodynamic compromise with hypovolemic shock after a fasting period that was necessary for an endoscopic exam. After this episode, he developed seizures and has since been prescribed sodium valproate.

\section{Molecular Analysis}

We examined the genomic DNA extracted from peripheral blood leukocytes. First, all patients were tested for mutations in the AVPR2 gene. Direct sequencing was performed, and mutations were found in all patients. The results are shown in Table 4. In patients with mutations in $A V P R 2$, their mothers were tested, and carrier status was confirmed in 4 of 5 cases.

Two unrelated children presented identical mutations (S167L) in the AVPR2 gene. Two patients had a mutation that has been described elsewhere in other Brazilian patients (R337X). The molecular analysis results are summarized in Table 4 .

Table 4 - Molecular results

\begin{tabular}{lccc}
\hline Patient & Gene & Mutation & Parents \\
\hline 1 & $A V P R 2$ & c.500C $>\mathrm{T}(\mathrm{S} 167 \mathrm{~L})$ & Heterozygous mother \\
2 & $A V R P 2$ & c.500C $>\mathrm{T}(\mathrm{S} 167 \mathrm{~L})$ & Heterozygous mother \\
3 & $A V P R 2$ & c.1009C $>\mathrm{T}(\mathrm{R} 337 \mathrm{X})$ & Heterozygous mother \\
4 & $A V P R 2$ & c.382T $>\mathrm{G}(\mathrm{Y} 128 \mathrm{D})$ & No mutation found \\
5 & $A V P R 2$ & c.1009C $>\mathrm{T}(\mathrm{R} 337 \mathrm{X})$ & Heterozygous mother \\
\hline
\end{tabular}

\section{DISCUSSION}

Hereditary NDI is a genetic condition characterized by an insensitivity of tubular cells to AVP despite normal or increased levels of this hormone. It is a severe disease, with manifestations in early infancy - including polyuria, polydipsia, hyposthenuria and hypernatremic dehydration. This disease can be life-threatening and is aggravated by feeding the child infant formula instead of breast-feeding due to its increased sodium content. The clinical suspicion is confirmed by the specific tests cited above.

We have observed improved growth and weight gain with adequate therapy, such as an increased water intake rate, under a low-salt diet in the context of hydrochlorothiazide administration, similar to that reported in Lejarraga et al. ${ }^{15}$

Treatment with thiazides, which promote decreased free water clearance by increasing both sodium reabsorption and water retention, is also thought to increase the luminal expression of AQP2 and distal renal sodium transporters. ${ }^{16}$ The use of non-steroidal anti-inflammatory drugs reduces the urinary volume. However, a possible side-effect is the reduction of the glomerular filtration rate, leading to chronic renal failure with low clearance over both medium- and long-term intervals. Patient 2 underwent this therapeutic option with successful reduction in the urinary volume, though he developed arterial hypertension which required discontinuation of the drug.

Adherence to treatment is essential for clinical recovery, especially in the X-linked cases of NDI. The NDI cases caused by $A Q P 2$ mutations are responsible for a more severe clinical picture and poor response to treatment. Finally, urinary tract dilatation and neurogenic bladder are not uncommon manifestations. Preventive techniques, such as frequent voiding and nocturnal catheterization, might be considered as options to avoid these complications.

Intracellular retention of a functional vasopressin V2 receptor (V2R) mutant is a major cause of congenital NDI, and the rescue of V2R mutants by nonpeptide antagonists may restore their basolateral membrane localization and function. ${ }^{3}$ The method by which CPAns (cell permeable antagonists) rescue V2R mutants has been the subject of several studies. ${ }^{12}$ At present, however, it is not completely clear which features of CPAn are important to give the best functional rescue of V2R mutants under such conditions. A crucial aspect necessary for functional rescue besides rescued cell surface expression of the mutant is displacement of the V2R-bound antagonist by AVP to generate a cAMP response. $^{13}$

Binding of CPAns to a mutant receptor can reverse the distorting effect of the mutation and thus aid in protein folding. Following rescue to the cell surface, however, most of the V2R mutants are able to bind AVP and consequently elicit a cAMP response. Functional rescue can only be achieved if the pharmacological chaperone is displaced by an agonist, thereby allowing receptor activation and induction of the signaling cascade. ${ }^{13}$

Therefore, to be of clinical value, the functional rescue of V2R mutants should occur at low concentrations of antagonists and AVP; in addition, the restoration of function should last as long as possible.

Recent studies with these antagonists have shown that the functional rescue of mutant V2Rs at clinically practical concentrations is most effective with high-affinity antagonists. Morello et al. showed that pretreatment with the high-affinity cell-permeable V2R antagonist SR121463A restored cell surface expression in 8 of 15 ER-retained V2R mutants that were subsequently activated by AVP (i.e., functional rescue). ${ }^{12}$ Recently, Robben et al. have demonstrated that the antagonists OPC31260 and OPC41061 promote a functional rescue of the V2R mutant. OPC 31260 is currently being tested as a treatment for polycystic 
kidney disease, ${ }^{17}$ while OPC41601 is under trial to treat hyponatremia and congestive heart failure in humans. ${ }^{18}$ Since negative side effects have not been reported in these studies, OPC31260 and OPC41061 represent safe and promising candidates to treat NDI in patients with V2R mutants. ${ }^{19}$ Certainly more studies are necessary to confirm their efficacy and lack of toxicity. In conclusion, the molecular defect characterization seems to be essential to best select potential patients to be treated with these pharmacochaperones.

In this study we detected two families (those of patients 1 and 2), apparently unrelated, who exhibited the same mutation in AVPR2 (S167L), as well as two other patients who presented a mutation that had been reported elsewhere in Brazil (R337X). ${ }^{9}$ This may raise the possibility of a founder effect in some Brazilian cases.

In addition, to illustrate the importance of molecular analysis in the context of genetic counseling, four of our five patients with AVPR2 mutations inherited the disease from their mothers. Only patient 4 showed a de novo mutation (Y128D) in AVPR2, since his mother's molecular analysis data was normal.

This study reported the clinical and laboratory characterization of NDI and emphasizes the importance of genetic investigations as a basis of disease diagnosis. Our work reiterates the need for genetic counseling for the affected families.

\section{REFERENCES}

1. Sasaki S. Nephrogenic diabetes insipidus: update of genetic and clinical aspects. Nephrol Dial Transplantation. 2004;19:1351-3.

2. Knoers NV, Deen PM. Molecular and cellular defects in nephrogenic diabetes insipidus. Pediatr Nephrol. 2001;16:1146-52.

3. Bichet DG. Nephrogenic Diabetes Insipidus. Advances in Chronic Kidney Dis. 2006; 13:96-194.

4. Morello JP, Bichet DG. Nephrogenic Diabetes Insipidus. Annu Rev Physiol. 2001; 63:607-30.

5. Schrier RW. Body Water Homeostasis: Clinical Disorders of Urinary Dilution and Concentration. JASN 2006;17:820-2.

6. Morello JP, Bouvier M, Petäjä-Repo UE, Bichet DG. Pharmacological chaperones: a new twist on receptor folding. Trends Pharmacol Sci. 2000;21:466-9.
7. van Lieburg AF, Verdijk MA, Schoute F, Ligtenberg MJ, van Oost BA, Waldhauser F, et al. Clinical phenotype of nephrogenic diabetes insipidus in females heterozygous for a vasopressin type 2 receptor mutation. Hum Genet. 1995;96:70-8.

8. Seibold A, Brabet P, Rosenthal W, Birnbaumer M. Structure and chromosomal localization of the human antidiuretic hormone receptor gene. Am J Hum Genet. 1992; 51:1078-83.

9. Boson WL, Della Manna T, Damiani D, Miranda DM, Gadelha MR, Liberman B, et al. Novel vasopressin type 2 (AVPR2) gene mutations in Brazilian nephrogenic diabetes insipidus patients. Gen Test 2006;10:157-62.

10. Fujiwara TM, Bichet DG. Molecular Biology of Hereditary Diabetes Insipidus. J Am Soc Nephrol. 2005;16:2836-46. 
11. Sasaki S, Fushimi K, Saito H, Saito F, Uchida S, Ishibashi K, et al Cloning, characterization, and chromosomal mapping of human aquaporin of collecting duct. J Clin Invest. 1994;93:1250-6.

12. Morello JP, Salahpour A, Laperriere A, Bernier V, Arthus MF, Lonergan $\mathrm{M}$, et al. Pharmacological chaperones rescue cell-surface expression and function of misfolded V2 vasopressin receptor mutants. J Clin Invest. 2000;105:887-95

13 . Robben JH, Sze M, Knoers NVAM, Deen PMT. Functional rescue of vasopressin $\mathrm{V} 2$ receptor mutants in MDCK cells by pharmacochaperones: relevance to therapy of nephrogenic diabetes insipidus. Am J Physiol Renal Physiol. 2007;292:F253-60.

14. Friedman E, Carson E, Larsson C, DeMarco L. A polymorphism in the coding region of the vasopressin type 2 receptor (AVPR2) gene. Hum Mol Genet. 1993; 2:1746.
15. Lejarraga H, Caletti MG, Caino S, Jimenéz A. Long-term growth of children with nephrogenic diabetes insipidus. Pediatr Nephrol. 2008;23:2007-12.

16. Kim GH, Lee JW, Oh YK, Chang HR, Joo KW, Na KY, et al. Antidiuretic Effect of Hydrochlorothiazide in Lithium-Induced Nephrogenic Diabetes Insipidus Is Associated with Upregulation of Aquaporin-2, $\mathrm{Na}-\mathrm{Cl} \mathrm{Co}$ transporter, and Epithelial Sodium Channel. JASN. 2004;15:2836-43.

17. Bennet WM. V2 Receptor antagonists in cystic kidney diseases: an exciting step towards a practical treatment. JASN. 2005;16:838-9.

18. Ohmishi A, Orita Y, Takagi N, Fujita T, Toyoki T, Ihara Y, et al. Aquaretic effect of a potent, orally, active, nonpeptide V2 antagonist in men. J Pharmacol Exp Ther. 1995;272:546-51.

19. Serradeil-Le Gal C, Wagnon J, Valette G, Garcia G, Pascal M, Maffrand JP, et al. Nonpeptide vasopressin receptor antagonists: development of selective and orally active V1a, V2 and V1b receptor ligands. Prog Brain Res. 2002;139:197-210. 